\section{$\underset{\substack{\text { hommes } \\ \text { \& migrations }}}{ }$}

Hommes \& migrations

Revue française de référence sur les dynamiques

migratoires

$1290 \mid 2011$

Travailleurs sociaux et migrations

D’une réalité de sans-papiers à une construction d'identité sociale complexe

\title{
Les trajectoires des jeunes migrants ou leur installation en France
}

\author{
Marie-José Pagnon
}

\section{(2) OpenEdition \\ Journals}

\section{Édition électronique}

URL : http://journals.openedition.org/hommesmigrations/745

DOI : 10.4000/hommesmigrations.745

ISSN : 2262-3353

\section{Éditeur}

Musée national de l'histoire de l'immigration

\section{Édition imprimée}

Date de publication : 1 mars 2011

Pagination : 88-96

ISSN : 1142-852X

Référence électronique

Marie-José Pagnon, «Les trajectoires des jeunes migrants ou leur installation en France », Hommes \& migrations [En ligne], 1290 | 2011, mis en ligne le 01 mars 2013, consulté le 19 avril 2019. URL : http:// journals.openedition.org/hommesmigrations/745; DOI : 10.4000/hommesmigrations.745 
D'une réalité de sans-papiers à une construction d’identité sociale complexe

\section{Les trajectoires \\ des jeunes migrants ou leur installation en France}

Par Marie-José Pagnon, conseillère en économie sociale et familiale

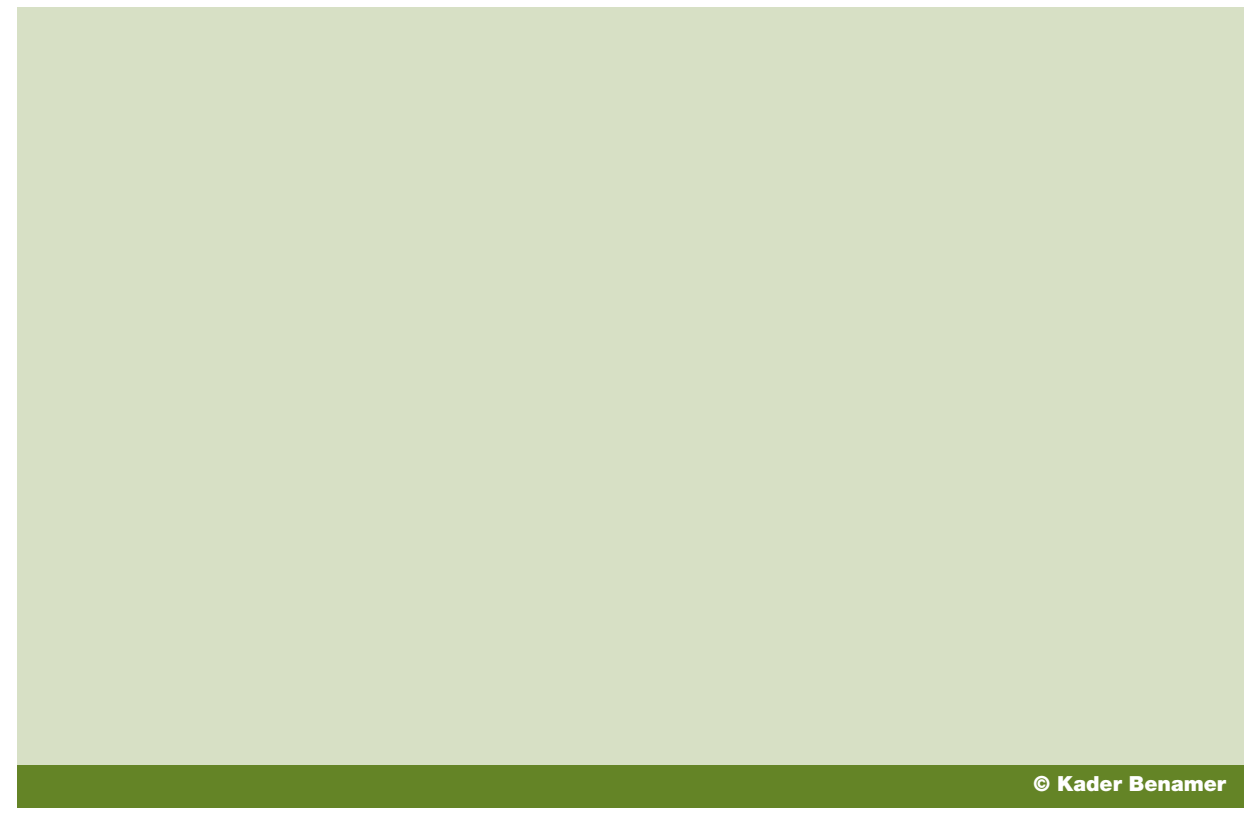

Le parcours des jeunes migrants étrangers en France est trop souvent évalué en fonction des seules raisons de leur départ et de la nécessaire intégration qui conditionne l'obtention de la nationalité française. Or, si la réussite de leur installation dépend des procédures législatives du pays d'accueil, elle est avant tout le fruit de stratégies individuelles diversifiées où la famille d'origine joue un grand rôle. Le "devenir français" répond au souhait de vivre en paix, sans pour autant se couper de ses racines culturelles. 
La migration des mineurs étrangers arrivant seuls en France date des années quatrevingt-dix. Peu de chiffres ont pu alors établir avec exactitude le nombre d'enfants concernés. Le rapport de l'Inspection générale des affaires sociales (IGAS) annonce 3100 mineurs admis par l'Aide sociale à l'enfance (ASE) en 2003, environ 2500 au 30 septembre 2004. Également en 2004, 1200 contrats jeunes majeurs ont été signés pour les jeunes migrants devenus majeurs. Il est certain que ce flux est inférieur à celui des personnes adultes, mais ce phénomène nouveau interroge les acteurs sociaux quant aux modes de prise en charge de cette population. Ces jeunes sont mineurs et relèvent donc de la protection de l'enfance, mais ils sont aussi étrangers "sans-papiers" et certains départements se sont questionnés quant à leur compétence en la matière. Linquiétude, émergeant du manque de savoir-faire à l'égard de cette population nouvelle pour les services de l'Aide sociale à l'enfance, s'est aussi transformée en appréhension quant à un éventuel coût exponentiel. Le rapport de l'IGAS note à ce sujet : "Selon les projections, le coût total des prises en charge se situerait dès lors entre 74 et 121 millions d'euros pour les 63 départements ayant répondu à l'enquête. Si on rapporte cette donnée au coût global de l'Aide sociale à l'enfance, on obtient une estimation de l'ordre de 1,6 à 2,6\%. Ce qui reste une proportion faible au regard du poids pris par la question des mineurs isolés dans les débats." Au-delà de ces données controversées sur la prise en charge de ces jeunes migrants, leur devenir est une préoccupation plus ancrée dans la réalité professionnelle des travailleurs sociaux. Un questionnement s'impose sur leurs conditions d' "installation" en France : chercher à comprendre comment ces jeunes migrants prennent place dans la société d'accueil, comment celle-ci leur offre une place, est plus fécond que de s'arrêter aux seuls chiffres. Autrement dit, il s'agit de saisir leurs trajectoires et d'analyser leurs stratégies en explorant et en questionnant les multiples manières dont ces jeunes vivent au sein de la société française.

Cette réflexion émane d'une observation de terrain en tant que professionnelle de l'Aide sociale à l'enfance intervenant auprès de jeunes de 17 à 21 ans. Elle s'est imposée lors du suivi des jeunes migrants dans le cadre de contrats jeunes majeurs. Car l'accompagnement social de cette population interroge et bouscule le travailleur social.

\section{Jeunes et étrangers : une double gageure pour l'action sociale}

Emmanuelle Santelli propose un autre regard sur les immigrés qu'un questionnement en termes d'intégration. Elle raisonne sur les manières de s'installer en France, cette pensée a la particularité de ne pas enfermer la population migrante dans un modèle mais au contraire de discerner la singularité de parcours complexes. Abdelmalek Sayad 
présente un développement des "trois âges de l'émigration". Un quatrième âge pourrait désormais représenter les nouvelles formes de migration fuyant la misère et la guerre, et se rapporter aux jeunes migrants arrivés seuls en Europe et appelés en France les "mineurs isolés étrangers (MIE)". Le devenir de ces jeunes étrangers est certainement plus préoccupant au quotidien du fait de leur statut juridique particulier et de l'absence de famille. De plus, l'accompagnement social a la particularité de se poursuivre parfois au-delà de la mission : l'âge du jeune met un terme au suivi social, alors que le statut juridique nécessite encore un soutien pour certaines démarches (auprès de la préfecture, par exemple).

Les jeunes migrants confiés à l'Aide sociale à l'enfance présentent des particularités identifiées dans le rapport IGAS. Ils sont différents du public accueilli habituellement à l'ASE : ils sont qualifiés de jeunes en forte demande d'intégration et ils ne relèvent pas de la même problématique "enfance en danger". On n'évoque pas pour eux le terme de "maltraitance familiale".

Lanalyse des modalités de construction des identités sociales de ces jeunes s'est construite au travers des trajectoires singulières de neuf d'entre eux. Ils ont entre 21 et 25 ans, sont d'origine étrangère (deux Albanais, dont un natif du Kosovo, trois Angolais, un Arménien, trois Congolais de la République démocratique du Congo, ex-Zaïre). Arrivés seuls sur le territoire français, alors âgés de 15 à 17 ans, "sans papiers", ils ont été confiés à l'Aide sociale à l'enfance. Cette observation de terrain a conduit à poser des hypothèses. L'absence de famille, le traumatisme de l'exil, la barrière de la langue, l'incertitude de l'avenir quant à un statut juridique, la compréhension de nouvelles normes sont autant d'éléments pouvant conduire ces jeunes migrants à l'échec. Et pourtant, ils s'installent selon un "modèle adulte" : ils vivent seuls ou en couple, avec parfois un enfant, ils ont suivi une formation et ils ont un travail. Ces jeunes semblent partir avec des "handicaps d'intégration forts" ; ils parviennent néanmoins à construire une trajectoire d'"émancipation"(famille et emploi). Sur quoi se fondent les trajectoires des jeunes migrants, quels sont les points de soutien, les moteurs, les héritages culturels, sur quelles solidarités s'appuient-ils pour construire leur parcours?

\section{Une situation juridique en évolution}

Une approche juridique est utile pour comprendre la situation des jeunes migrants interviewés. Elle est également nécessaire pour repérer les différents circuits de prise en charge des "mineurs isolés étrangers" qui, d'après le rapport IGAS, présentent "une forte hétérogénéité qui ne garantit pas une égalité de traitement sur le territoire". Ces commentaires laissent présager la disparité des chances en matière d'installation sur le territoire français. 
C'est donc par l'accès juridique que l'on peut tenter de comprendre comment les jeunes migrants prennent place dans la société d'accueil ; c'est ici saisir comment la société d'accueil leur offre une place. Cette approche juridique peut paraitre restrictive, dans le sens où elle ne présente que la situation de ces neuf jeunes pris en charge par le département de la Haute-Vienne entre 1999 et 2007.

Il convient de distinguer les changements intervenus après la loi du 26 novembre 2003. Comparer les parcours de ces jeunes migrants avant et après cette loi permet de différencier les manières de prendre place dans la société française puisque la loi a changé les modalités d'accueil de cette population. Il s'agit de montrer en quoi la loi participe ou pas au processus d'installation des jeunes migrants, car ils ne pourront s'installer que si une place leur est proposée. Avant 2003, la loi prévoyait que l'enfant confié aux services de l'Aide sociale à l'enfance peut, jusqu'à sa majorité, acquérir la nationalité française par déclaration devant

Le rapport nationalité française/installation s'est inversé : il faut aujourd'hui qu'un jeune migrant prouve son installation sur le territoire pour obtenir la nationalité française. le juge d'instance sans qu'il soit exigé de délai de prise en charge par les services de l'ASE. Cela résulte de la loi $\mathrm{n}^{\circ} 73-42$ du 9 janvier 1973, qui avait supprimé le délai de cinq ans de prise en charge institué au préalable. Les raisons conduisant la réforme de 1973 répondent à la volonté de placer les jeunes migrants privés de parents et ayant traversé de lourdes épreuves dans une situation favorable à une installation sur le sol français. L'attribution de la nationalité française est alors conçue non pas comme l'aboutissement d'un processus d'intégration, mais bien comme un moyen privilégié d'aide à l'installation et à trouver une place sur le territoire français.

Il est pertinent de réfléchir sur ce que peut provoquer le changement de nationalité. Lanalyse de Beate Collet permet d'apporter une réponse : "D’une manière générale, l'État français n'oblige pas à renoncer à la nationalité d'origine, ce qui signifie concrètement que les immigrés ou leurs enfants ne sont pas obligés de choisir entre leur nationalité d'origine et la nationalité française comme un conflit identitaire, mais peuvent la vivre comme une simple intégration politique, professionnelle et sociale, pour certains doublée d'une adhésion nationale plus soutenue."

Concrètement, sept jeunes interviewés ont déposé une demande d'acquisition de nationalité française auprès du tribunal d'instance, ils ont dû alors fournir des papiers d'état civil suffisamment clairs. Six jeunes ont obtenu la nationalité par ce biais-là. La loi n²003-1119 du 26 novembre 2003 relative à la maîtrise de l'immigration, au séjour des étrangers en France et à la nationalité (MISEFEN) a entraîné des 
modifications lourdes de conséquences quant à une possible installation des jeunes migrants. Cette loi réintroduit une condition de temps de prise en charge par l'ASE. C'est un bond en arrière de plus de trente ans que le législateur entreprend en notifiant cette condition de trois années. Pour les jeunes arrivés en 2003, il semblerait, à la lecture des dossiers, que l'application de la loi ait laissé peu de temps aux travailleurs sociaux pour être réactifs.

Deux jeunes n'ont plus satisfait les conditions subordonnées à l'obtention de la nationalité française. Ils ont été également tous les deux déboutés quant à leur demande d'asile. Ces jeunes interviewés bénéficient certes d'une carte de séjour, mais, d'une part, elle est temporaire et, d'autre part, elle ne s'appuie que sur un statut lié à une injonction d'activité, scolaire ou professionnelle. Le rapport nationalité française/installation s'est inversé : il faut aujourd'hui qu'un jeune migrant prouve son installation sur le territoire pour obtenir la nationalité française. Ces jeunes migrants sont liés à un devoir de réussite plus que tous les autres. Ils ont sans cesse une épée de Damoclès au-dessus de la tête, leur rappelant qu'ils sont étrangers majeurs et donc potentiellement "expulsables".

Les jeunes rencontrés lors de l'enquête sont maintenant en France depuis presque dix ans pour certains, et cinq ans pour les derniers arrivés. Des dispositions administratives permettant aux jeunes confiés aux services sociaux de disposer d'un titre de séjour régulier et pérenne devraient être méditées. Leur parcours laisse entrevoir une volonté d'émancipation dans un cursus d'installation sur le territoire français, même pour les jeunes les plus en difficulté quant à leur statut administratif encore précaire.

\section{Différents profils d’installation}

Certains ont donc obtenu la nationalité française. La question essentielle est de savoir si cela les a aidés à s'installer en France. La combinaison des conditions de départ et des modalités d'accueil complexifie les manières de s'installer et de prendre place dans la société française : on ne s'installe pas de la même manière dans un pays selon les circonstances du départ, selon l'histoire familiale et la poursuite ou non de ses liens, de ses attaches et de ses alliances. On s'installe aussi différemment selon l'accueil reçu. Avant 2003, il ne fallait pas apporter la preuve d'une "intégration réussie" pour obtenir la nationalité française. Cette réflexion amène à présenter une esquisse de quatre profils, en sachant que ce n'est pas neuf récits qui peuvent conduire à une typologie. 


\section{Premier profil : S'installer en France pour devenir un autre.}

Dans ce cas, l'acquisition de la nationalité française paraît salvatrice. Ce profil permet de comprendre la complexité des trajectoires, entre la contrariété d'une arrivée fortuite en France (l'un souhaitant aller en Angleterre et l'autre au Canada) et au final un arrangement avec l'acquisition d'une nouvelle nationalité. Celle-ci se substitue non pas à une "identité culturelle", mais à une "nationalité assignée" par un oppresseur. C'est le cas du jeune Albanais du Kosovo, de nationalité serbe, et du jeune Arménien né en Azerbaïdjan. Ce qui apparaît comme un compromis au départ (l'acceptation de rester en France procurant en échange une nouvelle nationalité) est de toute évidence un soutien parmi d'autres à une installation. Par ce biais, les jeunes se défont du poids d'"un habillage identitaire" lourd à porter par tout un peuple. Ils endossent alors une autre "enveloppe" qui n'entre pas en conflit avec le noyau culturel.

\section{Deuxième profil : S’installer en France pour sa famille.}

Les jeunes ont accepté la nationalité française comme une démarche administrative favorisant le retour dans leur pays d'origine et le contact avec leur famille, sans pour autant remettre en cause une identité composée de leurs racines et de leur vécu. Dans ce profil, les jeunes migrants ne semblent pas troublés par l'acquisition de la nationalité française, même s'ils la présentent comme une proposition émanant de tiers, en l'occurrence des travailleurs sociaux. Ce choix, qui au final leur appartient, est à mettre en perspective avec la possibilité d'un retour dans le pays d'origine et c'est cela qui confirme le bien-fondé de leur décision. Ils s'installent donc dans le pays d'accueil avec pour appui la légitimité d'un choix, et c'est certainement cette légitimité qui constitue un des soutiens à leur installation en France.

\section{Troisième profil : S’installer en France pour vivre en paix.}

Parmi les jeunes de l'échantillon, c'est dans cette représentation que la souffrance s'est le plus exprimée. Ils ont été témoins du meurtre de leurs parents, et la vision de la mort n'a cessé de les hanter. De toute évidence, l'obtention de la nationalité française leur a permis avant tout d'acquérir des papiers et les a aidés à se reconstruire. Peut-on alors parler de nouveau départ ? Dans ce profil, l'obtention de la nationalité française s'inscrit dans un processus de réassurance garantissant une vie en démocratie.

\section{Quatrième profil : Incertitudes sur l'installation.}

Les représentants de ce profil sont les seuls à ne pas avoir obtenu la nationalité française. Ces jeunes sont arrivés en France en 2003, l'année du changement de loi quant aux conditions d'obtention de la nationalité française pour les mineurs étrangers relevant de l'ASE. Il est vrai que l'incertitude a concerné tous les jeunes à leur 
arrivée, mais cinq ans après, ils sont seuls encore dans l'expectative d'un statut leur procurant une assurance de non-expulsion. Ce profil met en avant tout le désenchantement que les jeunes ont éprouvé vis-à-vis de l'acquisition d'un statut juridique leur permettant de séjourner en France. C'est dans la reconnaissance professionnelle qu'ils espèrent leur salut. Ils ont compris qu'ils étaient dans l'obligation de réussir, ils n'ont pas droit à l'erreur. Il faut donc qu'ils prouvent leur volonté d" intégration" pour obtenir le droit de rester en France.

\section{Le rôle de la famille : de l'absence physique au maintien de liens symboliques}

Les jeunes interviewés sont arrivés seuls sur le territoire français, mais ils ne sont pas dépourvus pour autant d'une mémoire familiale qui demeure pour l'essentiel le moteur d'une installation sociale et professionnelle. La famille, disparue ou encore présente dans le pays d'origine, accompagne la trajectoire professionnelle tout comme la trajectoire privée. La famille est toujours présente dans les récits, même si les histoires ne sont pas semblables. Elle a une place qui peut être le moteur essentiel autour duquel se forment les projets. Elle occupe également un rôle périphérique qui s'inscrit dans une transmission de valeurs même lorsqu'elle a disparu. La direction prise dans la sphère privée est empreinte du lien familial. Même décédée, la famille réapparait comme un fil conducteur.

Ces jeunes font référence à leur passé au travers des valeurs comme le respect et notamment celui envers la famille. Ils se sentent chargés d'une mission envers elle. Ils ont renoué des contacts lorsque les parents vivent encore dans le pays d'origine. Ils orientent leur trajectoire professionnelle de manière à leur apporter une aide. Lintérim n'est pas vécu comme une situation professionnelle précaire mais comme une stratégie pour être disponible envers ses proches. Ils adaptent leur projet avec la détermination d'assumer la famille d'origine ou la nouvelle famille qu'ils ont créée.

Les jeunes migrants interrogés ne se sentent pas "abandonnés" ou en conflit avec le milieu familial, comme les autres jeunes de l'Aide sociale à l'enfance. C'est cette relation à la famille qui les différencie des jeunes habituellement accueillis à l'ASE. Parmi les jeunes interviewés, certains sont en contact régulier avec leur famille. Être parvenu à quitter son pays d'origine, c'est peut-être avoir été protégé de la misère, de la guerre, de la souffrance par la famille. Cette dernière ne semble pas revendiquer quoi que ce soit, ni même le fait de venir en France. Ce projet semble davantage émaner du jeune. Cela met en évidence une autre manière de s'installer. Les jeunes migrants conçoivent alors l'éventualité d'une famille à nouveau réunie, mais 
cette fois en France. Le lien familial, qu'il soit encore très fort ou estompé, met en évidence dans le parcours des jeunes ce qu'Emmanuelle Santelli décrit comme "la confrontation, la négociation, l'accumulation des expériences, des transmissions, des aspirations...et ce, parce que chacun est à la fois produit et acteur de son histoire familiale". Leurs discours ne laissent pas transparaître une quelconque pression familiale mais plutôt une mémoire de l'aide reçue. Ils se missionnent, "ils s'auto-mandatent", comme l'a écrit Angélina Étiemble. Ces jeunes vivent leur départ comme une chance dont certains se sentent redevables.

S'ils conçoivent leur installation en France en se mêlant au paysage de la terre d'accueil,

\section{Le mérite individuel, peut conduire à sélectionner "les bons" des "mauvais" étrangers.} ils ont besoin de proximité avec leur culture où il est possible de faire peser leur identité. La communauté peut aussi être un relais et assurer une fonction intermédiaire nécessaire à un équilibre entre le pays d'origine et le pays d'accueil pour des jeunes migrants arrivés depuis peu en France. La dimension culturelle et religieuse peut constituer cet accès. La pratique de la religion occupe une place importante dans la vie de ces jeunes. C'est dans le lieu de culte qu'ils retrouvent des compatriotes. L'approche d'Emmanuel Jovelin conforte cette lecture : "Les liens sont maintenus aussi de manière symbolique (attachement à la religion d'origine, musique du pays d'origine, attention particulière quant à l'évolution de la situation du pays à travers des médias, etc.)." Il importe ici de comprendre comment les références à la communauté d'origine (qu'elles soient dans le choix du conjoint, des amis, mais aussi dans des pratiques culturelles, religieuses ou culinaires) participent au processus d'installation pour ces jeunes migrants arrivés seuls sur le territoire français. Une installation qui prend sens dans le pluralisme culturel.

\section{Penser l'installation en termes de trajectoires}

Selon Emmanuelle Santelli, "lintérêt à accorder à ces histoires et à ces transmissions familiales est grand et plus fécond que de considérer les trajectoires professionnelles 'réussies' comme étant le résultat du mérite individuel et lou de l'efficacité du modèle républicain". Le terme "émancipatrices" sera préféré à "réussies" pour qualifier les trajectoires de ces jeunes migrants. Ce n'est surtout pas le résultat du mérite individuel qui doit être abordé dans les trajectoires, mais plutôt des histoires de vie où la mémoire familiale sert de "guidance". Le mérite individuel peut conduire à sélectionner "les bons" des "mauvais" étrangers. 
Peu importe de vérifier si un jeune est intégré. Considérer l'efficacité du modèle républicain reviendrait à ne pas prendre en compte dans l'analyse des trajectoires tout ce qui nous paraît essentiel à la construction des identités sociales de ces jeunes, à savoir le rôle et la place de la communauté d'origine.

Réfléchir sur les manières de prendre place dans la société française, autrement dit de s'installer, c'est décortiquer le parcours des jeunes migrants en référence à ce qui relève de la socialisation primaire, la famille, pour arriver à la construction d'une vie de couple, item de la socialisation secondaire. Cette même configuration se retrouve au niveau des études et de l'emploi. Ce cheminement met en exergue la trajectoire des jeunes dans une perspective d'évolution où le jeune étranger a la capacité de développer des stratégies. L'installation des jeunes migrants selon la trajectoire familiale/matrimoniale et la trajectoire scolaire/professionnelle permet d'avoir une lecture favorisant les allersretours entre le pays d'origine et le pays d'accueil dans la parole des jeunes interviewés. Ils font référence aux raisons pour lesquelles ils sont partis de leur pays d'origine, aux causes pour lesquelles ils y retournent ou pas, à la culture familiale et ethnique qui les a nourris. Ces références s'aménagent avec ce qu'ils construisent dans le pays d'accueil, et c'est cette combinaison entre pays d'origine et pays d'accueil qui participe à l'élaboration des trajectoires. Analyser les trajectoires sociales des jeunes à partir de l'axe scolaire/professionnel et de l'axe familial/matrimonial permet d'articuler les différentes formes de socialisation acquises du pays d'origine au pays d'accueil.

\section{Conclusion}

Cette approche apporte peut-être une autre dimension dans la recherche sur les jeunes migrants. Ils sont habituellement étudiés dans leur parcours migratoire, c'est-à-dire en prenant en compte essentiellement les raisons de leur arrivée et non en suivant leur installation repérée à partir d'un nombre d'années passées en France. Cette démarche s'inscrit dans une volonté de susciter un regard différent sur ces jeunes : au lieu de les poser en victimes de flux migratoires, il s'agit de les montrer acteurs de leur destinée. En faisant ce travail, il est bien évident que la posture que revêt "l'apprenti chercheur" n'est pas neutre dans un tel contexte. Pour avoir entendu trop de discours suspicieux à l'égard de ces jeunes (faux papiers d'identité, tricherie sur l'âge réel...), c'est en tant que professionnelle, mais aussi en tant que militante et "apprentie chercheuse" que cette question de l'installation des jeunes migrants s'est imposée. De plus, le désir de marcher sur les pas de Gérard Noirel s'est manifesté, notamment à la lecture de cette phrase : "Je souhaitais par-dessus tout contribuer à faire reculer les préjugés et les fantasmes qui ont jalonné l'histoire de l'immigration depuis le XIX siècle", mais il ajoute: "Malheureusement, je suis bien obligé de reconnaître que le but n'est pas atteint." 